\title{
Effectiveness of Life Style Modification on Lipid Profile for Patients with Hyperlipidemia
}

\author{
Magda Moawad Mohsen ${ }^{1}$, Neima Ali Riad ${ }^{2}$, Amina Ibrahim Badawy ${ }^{2, *}$, \\ Badria Mahrous Abd El-Hammed ${ }^{2,3}$, Dalia Mahmoud Abd Elmonem Elsherbini: ${ }^{4} 5$ \\ ${ }^{1}$ Community Health Nursing, Faculty of Nursing, Menoufia University, Al Minufya, Egypt \\ ${ }^{2}$ Medical-Surgical Nursing, Faculty of Nursing Menoufia University, Al Minufya, Egypt \\ ${ }^{3}$ Nursing Department, Faculty of Applied Medical Science, Al Baha University, Al Baha, Saudi Arabia \\ ${ }^{4}$ Department of Clinical Laboratory Sciences, College of Applied Medical Sciences, Jouf University, Sakaka, Saudi Arabia \\ ${ }^{5}$ Department of Human Anatomy and Embryology, Faculty of Medicine, Mansoura University, Mansoura, Egypt \\ Email address: \\ amina73737373@yahoo.com (A. I. Badawy) \\ ${ }^{*}$ Corresponding author
}

\section{To cite this article:}

Magda Moawad Mohsen, Neima Ali Riad, Amina Ibrahim Badawy, Badria Mahrous Abd El-Hammed, Dalia Mahmoud Abd Elmonem Elsherbini. Effectiveness of Life Style Modification on Lipid Profile for Patients with Hyperlipidemia. American Journal of Nursing Science. Vol. 9, No. 1, 2020, pp. 8-18. doi: 10.11648/j.ajns.20200901.12

Received: December 9, 2019; Accepted: December 23, 2019; Published: January 4, 2020

\begin{abstract}
Background: Major predisposing factors of Coronary artery diseases were urbanized lifestyles and growth of ageing populations in developing country like Egypt. The modifiable risk factors for cardiovascular disease (CVD) such as hyperlipidemia, diabetes mellitus, and obesity are likely to increase in the future, but they can be controlled through lifestyle modification. Aim of the study: This study was carried out to examine the effect of life style modification for patients with hyperlipidemia on their lipids profile. Design: Quasi experimental design was utilized. Setting This study was conducted among the patients attending at cardiac outpatient department, Menoufia University Hospital, Menoufia Governorate, Egypt. Subject: A purposive sample of 100 men and women attending cardiac outpatient clinic were selected. The subjects were divided equally into study and control groups. Instrumentations: a. Structured interview questionnaire: it consists of sociodemographic and medical data b. Bio-physiological measurement: Fasting venous blood samples for Glycosylated hemoglobin level, Lipid profile were obtained from all participants, and sent to biochemistry laboratories. Blood pressure assessment and BMI for all patients were compiled. Results: Statistical analysis showed that there were statistical differences among study and control group regard to total cholesterol, triglyceride, high-density lipoprotein cholesterol and low-density lipoprotein cholesterol and their arterial blood pressure level. Conclusion: The need for tailoring and following up of intervention for coronary artery diseases is an essential maneuver to reduce their risk factors occurrence. Recommendations: Implementation of lifestyle modification for at risk patient for coronary artery diseases by modifying their lifestyle. Routine screening for all subjects above the age of forty to detect abnormal lipid profile early and to increase their awareness to prevent and control of cardiovascular disease (CVD) is recommended.
\end{abstract}

Keywords: Life Style Modification, Lipid Profile, Hyperlipidemia

\section{Introduction}

Hyperlipidemia is an excess of lipids in the blood, largely cholesterol and triglycerides. It can be manifested by excess of the serum total cholesterol, low-density lipoprotein (LDL) cholesterol and triglyceride concentrations, and a decrease in the high-density lipoprotein (HDL) cholesterol concentration
$[1,2]$.

Hyperlipidemia is a common risk factor for the development of cardiovascular disease, there are many risk factors, and some can be controlled but not others. The risk factors that can be controlled (modifiable) are: hypertention, smoking; diabetes; overweight or obesity; sedentary life; unhealthy diet and stress. biochemical risk factors were 
increased levels of TG, total cholesterol, LDL-cholesterol, and low HDL-cholesterol level (El-Moselhy et al, 2018) [3, 4]. Those that cannot be controlled (conventional) are: Age (simply getting older increases risk); sex (men are generally at greater risk of coronary artery disease); family history; and race $[5,6]$.

World Health Organization (WHO) estimates suggested that $30 \%$ (17.3 million) of all deaths worldwide could be attributed to cardiovascular disease (CVD) [7, 8].

By the year 2030, the Goals of United Nation are reducing premature mortality from non-communicable diseases by a third especially Cardiovascular diseases which are the most common non-communicable diseases globally and they are responsible for an estimated $17 \cdot 8$ million deaths in 2017 , of which more than three quarters were in developing countries [8]. By 2025, adults aged 40 years or older who are at high risk of cardiovascular disease will be reduced for at least $50 \%$ by providing teaching and drug treatments based on WHO recommendation to reduce the global burden of cardiovascular disease [9].

Lifestyle modification involves changes for long-term habits and maintaining the new behavior for months or years. Lifestyle modification includes, apart from what we eat and drink, physical activity, weight reduction, smoking, and stress. Based on the recommendation of the WHO, stated that the all health facilities should enhance health promotion activities by creating an environment conducive to therapeutic lifestyle change (TLC) regarding diet, weight management, increasing physical activity and smoking cessation for the purpose of reduction the morbidity and mortality of CAD. [10, 11]. Diet teaching should be emphasized on minimizing intake of highly saturated fat and cholesterol. Weight reduction and encouraging physical activity should be promoted following Body Mass Index (BMI) targets and WHO recommendations for physical activity. A study conducted among elderly Egyptian patients with $\mathrm{CAD}$ and concluded that modifiable risk factors that can have an effective role in preventing and better treatment of CVD, and promoting patients health. This could be achieved through health education programs targeting the modifiable risk factors; smoking, obesity, sedentary lifestyle, bad dietary habits and soul and mind peace, and to trained patients regarding these lifestyle changes [12]. As indicated from figure 1. Lifestyle changes for preventing cardiovascular disease include physical activity, weight management, lipid modification therapy, diet management, and stopping smoking and alcohol. [12].

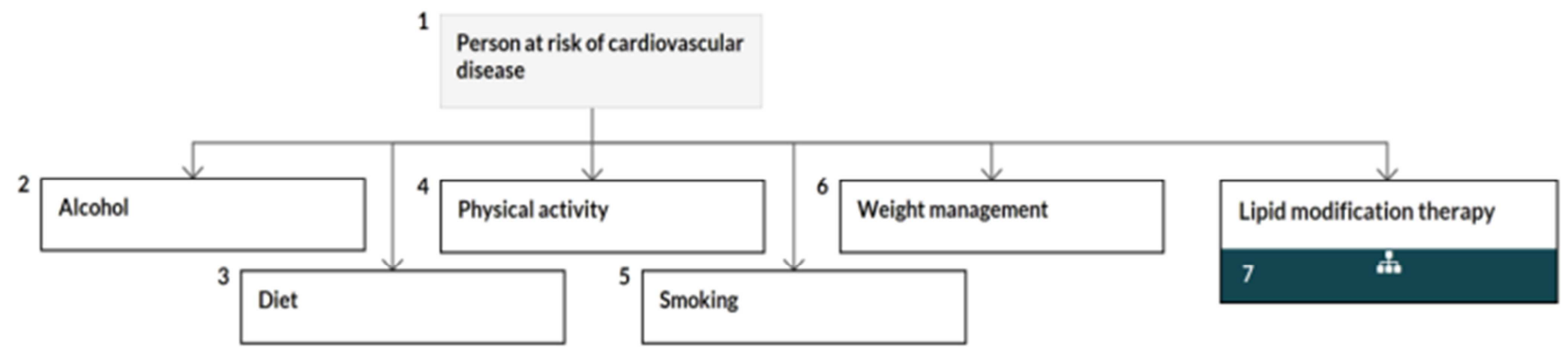

Figure 1. Lifestyle changes for preventing cardiovascular disease [12].

The recently published recommendations by The American College of Cardiology (ACC) and the American Heart Association (AHA) which are concerning lifestyle to reduce the risk of cardiovascular disease (CVD) addressed diet and nutrition, exercise and physical activity, body weight, tobacco use, as well, the guidelines concerning dietary recommendations such as eating a diet high in vegetables, fruit, legumes, nuts, whole grains, and fish, limiting sodium, saturated fats, refined carbohydrates, sweetened beverages, and processed meats, and omitting trans fats [12]. lifestyle changes can reduce the risk of premature death and disability due to heart disease, both of diabetes and elevated level of cholesterol added as leading causes of increased incidence of CAD which still remains as one of the most leading causes of morbidity and mortality in Egypt where latest statistics in 2013, it is considered first killer [12-14].

Obesity frequently raises cholesterol levels in both verylow-density lipoprotein (VLDL) and LDL fractions, elevates triglyceride levels, lowers HDL cholesterol levels, raises blood pressure and promotes glucose intolerance. Weight loss lowers blood pressure and improves glycemic control, in addition to lowering total cholesterol and its LDL and VLDL fractions, triglycerides and raises HDL cholesterol [14-16].

Exercise programs are very important for patients to help tilt the caloric balance toward a caloric deficit and thus elicit weight loss. Aerobic exercise increases metabolic rate over the 24 hours post-exercise. Physical activity has multiple benefits that include increasing in bone density, support for and creation of new vascular tissue, increases in fat metabolism, insulin sensitivity, memory and brain function and providing the greatest increases in cardiorespiratory fitness and short-term weight loss. Up-dated studies have documented that exercise stimulates local cardiomyocyte stem cells and cardiomyocyte progenitor cells to increase proliferation and specialization to a greater extent in the heart. This finding is critical to patients who have experienced some forms of ischemic heart failure which are manifested by cardiac cell death. In regard to exercise impact on lipid profile, claimed that exercise leads to increase in HDL cholesterol and decrease in TGs level while dietary approaches eventually lead to decrease in TGs levels, LDL cholesterol, total serum cholesterol. Added the exercise that benefit from aerobic exercise that targets large muscle groups, performed for 30 minutes four or more times a week, 
overweight patients should engage in low-intensity exercise more frequently and for longer durations [17-20].

Dietary modification to reduce cardiovascular disease (CVD) risk remains the cornerstone of both the National Cholesterol Education Program (NCEP) and American Heart Association (AHA) recommendations for the treatment of hyperlipidemic individuals [21-23].

The diet for healthy heart should focuses on fiber intake from whole grains, vegetables, fruits, fish, poultry, and nuts, while curbing sugary foods and beverages to lower cholesterol levels by ten percent [24].

Other lifestyles of concern in managing CAD include smoking, alcohol and consumption. As lifestyle pattern influences the condition of CAD patients, it is important to find dietary habits and other lifestyle patterns for a good lipid profile [25] mentioned that combination of both of healthy diet and physical activity are effective pathway for the reduction of chronic disease and they have beneficial effects on the cardiovascular system. [26] study concluded that, daily intake of $1500 \mathrm{mg}$ of vitamin $\mathrm{C}$ has positive lowering effect on anthropometric measurements and lipid profile among obese patients. Vitamin $\mathrm{C}$ administration may be helpful in controlling obesity and related disorders by advising high intake of vitamin $\mathrm{C}$ through the diet like guava, citrus fruits etc., [26] added the intakes of vitamin D3, high protein, omega-3 fatty acid, calcium, high fibre diet, -glucan, zinc and multi-mineral/vitamin improved lipid profile. Seaweeds, nuts, mate tea, soy and argon oil intakes also ameliorated lipid profile. Concluded that prescription of exercise and nutrition by primary care providers may elicit greater long term weight loss than current medical weight management practices. Finally diet and exercise can have a beneficial effect on managing serum levels of lipid profile [26, 27].

\subsection{Significance of the Study}

It is suspected that lifestyle modification that can be administered though cardiac outpatient department in addition to usual care results in sustained lowering of cardiovascular risk in terms lowering $\mathrm{BMI}$, controlling $\mathrm{BP}$, controlling of $\mathrm{HbA1} \mathrm{c}$ values to an acceptable level of glycemic control as well as sustains a desirable lipid profile. In Egypt, a little nursing research had conducted to investigate the effect of life style modification among those patients. Therefore, there is a standing need to provide information about lifestyle modification for this category of patients, which might be useful to nursing as well as other health care professionals. Such intervention is an essential prerequisite for the alleviation of the disease risk factors. It is also hoped that this effort will allow those patients to assume an active, integral and participatory role in their health care, which is considered the utmost goal of any nursing intervention. Moreover, it might generate an attention and motivation for further researches into this area.

\subsection{Aim of the Study}

This study was carried out to examine the effect of life style modification for patients with hyperlipidemia on their lipid profile.

\subsection{Research Hypothesis}

1. The BMI of the patients who undergo intervention will show more improvement than who do not.

2. The blood pressure of the patients who undergo intervention will show more improvement than who do not.

3. The blood glucose level of the patients who undergo intervention will show more improvement than who do not.

4. The mean lipid profile score of the patients who undergo intervention will show more improvement than who do not.

\subsection{Operational Definitions}

Lifestyle: In sociology, a lifestyle is the way a person lives; in public health, lifestyle generally means a pattern of an individual's behavioral choices and practices that lead to elevated or reduced health risk [11].

Lifestyle modification is the adoption and practice of attitudes and habits (healthy behaviors) that promote healthy living and enhance wellness. Healthy living refers to regular display of healthy behaviors leading to promotion of optimum health of an individual [12].

\section{Subjects and Method}

\subsection{Study Variables}

The independent variable in the study was life style modification provided for the hyperlipidemic patients, while the dependent variable was lipid profile result and blood pressure, blood glucose result and body mass index.

\subsection{Design}

Quasi experimental design was utilized.

\subsection{Setting}

This study was conducted among the patients attending at cardiac out patients department Menoufia University Hospital, Menoufia Governorates, Egypt.

\subsection{Subject}

A purposive sample of 100 men and women attending cardiac outpatient department were selected. The subjects were divided equally into study and control groups, 50 subjects for each with the following matching criteria:
a) Age above 20 years old.
b) Blood pressure above $130 \backslash 90 \mathrm{mmHg}$ and
c) High lipid profiles results.

\subsection{Calculation of Sample Size and Power of the Study}

In order to calculate the required sample size, the 
researcher used the Epi statistical program from the Open Source Statistics for Public Health. The assumptions were: a two sided confidence level of $95 \%=(1-\alpha)$; a power $(1-\beta)$ or (\% chance of detecting) of $80 \%$; ratio of sample size, unexposed (control)/ exposed (study group) $=1 \%$ of unexposed with outcome (awareness) $=5 \%$; Then the researcher entered one of four parameters which was $\%$ of exposed, $\mathrm{s}=25 \%$, and the other three parameters would be calculated by the Epi website program and results were presented using methods of Kelsey and Fleiss (2010) [28] with a continuity correction. The final sample consisted of one hundred subjects.

\subsection{Instrumentation}

\subsubsection{Structured Interview Questionnaire}

It consists of sociodemographic and medical data. It included patient age, sex, level of education, occupation and medical history.

\subsubsection{Bio-physiological Measurement}

Fasting venous blood samples were obtained from all participants, and sent to biochemistry laboratories. Glucosylated hemoglobin (HbA1c) and Lipid profile assessment: lipoprotein and glucose was assessed by Cobas $\mathrm{C}$ 311 analyzer operation's manual from the clinical chemistry analyzer Cobas c311 5th generation. The lipid profile tests including total cholesterol (TC), triglyceride (TG), highdensity lipoprotein cholesterol (HDL-C) and low-density lipoprotein cholesterol (LDL-C).

\subsection{Procedure for Data Collection}

\subsubsection{Period of the Study}

The study was conducted starting from March 2019 to the end of October at the same year.

\subsubsection{Approval to Conduct the Study}

An official permission was obtained from director of Menoufia University Hospital and head nurses of the cardiac outpatient clinic after explanation the aim of the study.

\subsubsection{Protection of Human Rights}

Each subject was informed about the purpose and the nature of the study. The subject was informed that their participation is totally voluntary and the confidentiality and anonymity were assured.

\subsubsection{Ethical Approval Was Obtained}

Ethical approval was obtained written consent to participate in the study was obtained from all participants.

\subsubsection{Tool Validity}

The tools were tested for content validity by two expert in medical surgical nursing to ascertain relevance and competence

\subsubsection{Reliability}

Reliability of the tools was done by test- retest for measuring internal consistency with a period of two weeks interval. The cronback's alpha for the structured interviewing questionnaire tool was 0.89 indicating good reliability. The test and retest reliability of the biophysiological measurement was 0.91 indicating good reliability.

\subsubsection{Pilot Study}

Pilot study was done on a sample of ten hypertensive patients who attended the medical outpatient clinic. The aim was to test the feasibility of the study tools. According to the results obtained, some questions were restructured and rephrased to give the most accurate response.

1. A list of names of eligible subjects was obtained from the registered nurse of cardiac outpatient clinic to obtain base line data for the study subjects then subjects were randomly assigned to the study and control group.

2. Initially, the base line data about demographic and medical problems were collected. Also the base data of the blood pressure, body mass index, glycosylated hemoglobin (Hemoglobin1c) and lipid profile were measured.

The subject was reminded about following life style modification through phone once a week.

\subsubsection{The Lifestyle Modification (LSM)}

Teaching was given individually about the life style modification (LSM) for 8 weeks. At the counseling, a brochure containing information on exercise and diet were distributed. The patients were asked to do brisk walking exercises for at least 30 minutes four times per week. They were asked to follow diet schedule during the period of the study. The subject was reminded about following lifestyle modification through phone once a week. The patients were asked to come for measure blood pressure, BMI, lipid profile, and glycosylated hemoglobin (Hemoglobin1c) checkup (posttest) after 3 and 6 months period.

\subsubsection{Evaluation}

Evaluation was done at 3 and 6 months after the intervention, the blood pressure, body mass index, glycosylated hemoglobin (Hemoglobin1c) and lipid profile were measured.

\section{Statistical Analysis}

The data was tabulated and analyzed by using SPSS program (statistical package for social science software) version 18 .

Quantitative data was expressed as mean and standard deviation $(x \pm \mathrm{SD})$. A qualitative data was expressed in numbers and percentage (No. \& \%). T test and the relationships among quantitative variables, and one-way ANOVA, Statistical significance was started at p-value $<0.05$.

\section{Results}

Table 1 Participants in study and control group were on 
mean average $59.91 \pm 10.52$ and $57.01 \pm 9.50$ years old respectively, with nearly $76 \%$ and $60 \%$ of participants being female in both groups. Most participants (48\% and $44 \%$ ) were having a secondary school respectively. So, it was apparent that there are similarity and no significant difference between both in regard to all socio-demographic variables.

Table 2 shows that most of the Menoufia university cardiac outpatient department subjects had myocardial infarction (62\%), diabetes mellitus $(56 \%)$, nearly equal percentage had a history of hypertension (40\%) and heart failure $(42 \%)$, about one third of the total subjects had angina pectoris $(30 \%)$ and only $(20 \%)$ were smokers. Patients with an abnormal triglycerides level were more liable to suffer from coronary artery disease (CAD), where its percentage was $(71 \%)(70 \%)(90 \%)(82.1 \%)(85.7 \%)$ and it was considered as a paramount risk factor, followed by HDL-C then LDL-C among those patients

Table 3 reveals that BMI of the study group statistically significantly $(\mathrm{P}=0.05)$ decreased when comparing baseline assessment with the third assessment time. Only at third assessment (at 6 months of intervention), there were statistically significant differences both of intervention and control groups $(\mathrm{P}=0.05)$ where there was a decrease in BMI when compared intervention group with control group $(24.0+4.61$ vs. $28.3+4.81$ respectively). So, the first hypothesis was supported.

Table 4 reveals a highly statistically significant differences in systolic blood pressure in the study group $(\mathrm{p}=<0.001)$ and only a statistically significant differences in diastolic blood pressure $(\mathrm{p}<0.05)$ within the intervention group. Additionally this table reveals that there is a highly statistically significant difference $(p=0.003)$ between both groups at 6 months respectively after the intervention. It is apparent that levels and scores of arterial blood pressure of the intervention group were lower than those of the control group after receiving risk reduction intervention combining with the routine health education. So, the second hypothesis was supported.

Table 5 summarized the mean HbAlc values for both groups, first measurement of both study and control group individuals' mean HbA1c values showed a poor level of glycemic control, and no significant difference was found between the two groups. However, final measurement of the intervention group individuals' mean HbA1c values is $7.21+4.61 \mathrm{mg} / \mathrm{dL}$, which is considered as an acceptable level of glycemic control. The difference between the two groups for the final measurement was statistically significant.

So, the third hypothesis was supported.

Table 6 shows that highly statistically significant differences were found between intervention and control groups at second and third measurement for all lipid profile variables except for HDL-C $(\mathrm{mg} / \mathrm{dl})$ variable, where there is no statistical significant differences between both groups $(\mathrm{p}=0.61, \mathrm{p}=0.35, \mathrm{p}=0.31)$ respectively. likely, for the intervention group, the table also shows that the means of all lipid profile variables were improved all through study phases except for HDL-C (mg/dl) variable, This improvement was significantly associated with adherence to suggested lifestyle pattern, whereas, concerning the control group ones who only received a routine care, there were no statistically significant differences found for all lipid profile variables among the three measurements. So, the fourth hypothesis was supported.

Figure 2 shows the effect of age on lipid profile in male cardiac patients. Among male cardiac patients, the lipid values of the different age groups differed significantly for all TG, HDL-C, LDL-C and TC without increasing without advancing of age.

Figure 3 revealed effect of age on lipid profile in female cardiac patients. Among female cardiac patients, the lipid values of the different age groups differed significantly for all, TG, HDL-C, LDL-C and except TC level still stationary in all age groups.

Figure 4 clarified effect of educational level on lipid profile in cardiac patients.

In regard to the effect of educational level, it was appearing that subjects with primary level suffer from elevated level of TG, HDL-C, LDL-C while Cholesterol still stationary while subjects with secondary and university level had the same level in all lipid profile.

Table 1. Descriptive Characteristics of study and Control Groups pre-intervention.

\begin{tabular}{|c|c|c|c|c|c|}
\hline \multirow{2}{*}{ Variables } & \multicolumn{2}{|c|}{ Intervention Group $\mathbf{N}=\mathbf{5 0}$} & \multicolumn{2}{|c|}{ Control Group $\mathbf{N}=\mathbf{5 0}$} & \multirow{2}{*}{ P value } \\
\hline & No & $\%$ & No & $\%$ & \\
\hline \multicolumn{6}{|l|}{ Age } \\
\hline$<40$ years & 12 & 24 & 10 & 20 & \multirow{4}{*}{$0.34 \mathrm{~ns}$} \\
\hline $40-60$ years & 22 & 44 & 28 & 56 & \\
\hline$>60$ years & 16 & 32 & 12 & 24 & \\
\hline Mean \pm SD & 59. & & 57. & & \\
\hline \multicolumn{6}{|l|}{ Gender } \\
\hline Female & 38 & 76 & 30 & 60 & \multirow[t]{2}{*}{$0.71 \mathrm{~ns}$} \\
\hline Male & 12 & 24 & 20 & 40 & \\
\hline \multicolumn{6}{|c|}{ Levels of education } \\
\hline Primary & 16 & 32 & 10 & 20 & \multirow{3}{*}{$0.42 \mathrm{~ns}$} \\
\hline Secondary & 24 & 48 & 22 & 44 & \\
\hline University & 10 & 20 & 18 & 36 & \\
\hline
\end{tabular}


Table 2. Relation between Abnormal Lipid Profile and Coronary Artery Disease and its Association with Risk Factors.

\begin{tabular}{|c|c|c|c|c|c|c|c|c|}
\hline \multirow{4}{*}{$\begin{array}{l}\text { Coronary artery disease } \\
\left.\text { parameter (total } N_{.}=100\right)\end{array}$} & \multicolumn{8}{|c|}{ Lipid profile as a risk factor. } \\
\hline & \multicolumn{2}{|c|}{$\begin{array}{l}\text { TC Reference range } \\
0.00-200\end{array}$} & \multicolumn{2}{|c|}{$\begin{array}{l}\text { TG Reference range } \\
0.00-150\end{array}$} & \multicolumn{2}{|c|}{$\begin{array}{l}\text { LDL-C Reference range } \\
100-130\end{array}$} & \multicolumn{2}{|c|}{$\begin{array}{l}\text { HDL-C Reference range } \\
40-50\end{array}$} \\
\hline & Normal & Abnor. & Normal & Abnor. & Normal & Abno. & Normal & Abnor. \\
\hline & No. $(\%)$ & No. $(\%)$ & No. $(\%)$ & No. $(\%)$ & No. $(\%)$ & No. $(\%)$ & No. $(\%)$ & No. $(\%)$ \\
\hline Myocardial infarction $n=62(62 \%)$ & $34(54.8 \%)$ & $28(45.2 \%)$ & $18(29 \%)$ & $44(71 \%)$ & $30(48.4 \%)$ & $32(51.6 \%)$ & $26(41.9 \%)$ & $36(58.1 \%)$ \\
\hline Angina pectoris $n=30(30 \%)$ & $18(60 \%)$ & $12(40 \%)$ & $12(40 \%)$ & $18(60 \%)$ & $16(53.3 \%)$ & $14(46.7 \%)$ & $4(3.3 \%)$ & $26(86.7 \%)$ \\
\hline Smoking $\mathrm{n}=20(20 \%)$ & $14(70 \%)$ & $6(30 \%)$ & $6(30 \%)$ & $14(70 \%)$ & $10(50.0 \%)$ & $10(50.0 \%)$ & $8(40 \%)$ & $12(60 \%)$ \\
\hline Diabetes mellitus $n=56(56 \%)$ & $26(46.4 \%)$ & $30(53.6 \%)$ & $10(17.9 \%)$ & $46(82.1 \%)$ & $28(50.0 \%)$ & $28(50.0 \%)$ & $24(50 \%)$ & $28(50 \%)$ \\
\hline Heart failure $n=42(42 \%)$ & $18(42.9 \%)$ & $24(57.1 \%)$ & $6(14.3 \%)$ & $36(85.7 \%)$ & $20(47.6 \%)$ & $22(52.9 \%)$ & $22(52.4 \%)$ & $20(47.6 \%)$ \\
\hline
\end{tabular}

Table 3. Effect of lifestyle modification on BMI for study and Control Groups throughout Study period.

\begin{tabular}{lll}
\hline \multirow{2}{*}{ BMI at different points of assessment } & Study Group N=25 & Control Group N=25 \\
\cline { 2 - 3 } & Mean \pm SD & Mean \pm SD \\
\hline Baseline assessment (before intervention) & $31.1+6.98$ & $29.90+4.94$ \\
Second assessment (at 3 months of intervention) & $28.70+4.81$ & $29.0+4.35$ \\
Third assessment (at 6 months of intervention) & $24.0+4.61$ & $28.3+4.81$ \\
F - p value & $0.05^{*}$ & 0.31 \\
\hline
\end{tabular}

ns $=$ not significant $*=$ significant $>0.05$.

Normal (18.5-24.99).

Overweight (25-29.99).

Obese $(>30)$.

Table 4. Effect of lifestyle modification on Improving Systolic and Diastolic Pressure for cardiac patients throughout Study Period.

\begin{tabular}{|c|c|c|c|}
\hline \multirow{2}{*}{ Systolic and Diastolic pressure } & Study Group N=50 & Control Group $\mathbf{N}=\mathbf{5 0}$ & \multirow{2}{*}{ P value } \\
\hline & Mean \pm SD & Mean \pm SD & \\
\hline \multicolumn{4}{|c|}{ - Systolic blood pressure levels at different points of assessment } \\
\hline Baseline assessment (before intervention) & $157.4 \pm 11.7$ & $154.5 \pm 10.4$ & 0.43 \\
\hline Second assessment (at 3 months of intervention) & $143.5 \pm 9.9$ & $151.2 \pm 10.0$ & $0.02 *$ \\
\hline Third assessment (at 6 months of intervention) & $130.2 \pm 8.2$ & $151.3 \pm 10.0$ & $0.003 * *$ \\
\hline f-p value & $<0.001 * * *$ & $0.41 \mathrm{~ns}$ & \\
\hline \multicolumn{4}{|c|}{ - Diastolic blood pressure levels at different points of assessment } \\
\hline Second assessment (at 3 months of intervention) & $86.7 \pm 7.7$ & $88.4 \pm 7.7$ & 0.31 \\
\hline Third assessment (at 6 months of intervention) & $81.3 \pm 7.3$ & $89.0 \pm 7.9$ & $0.02^{*}$ \\
\hline$f-p$ value & $<0.05^{*}$ & $0.46 \mathrm{~ns}$ & \\
\hline
\end{tabular}

Table 5. Effect of Lifestyle Modification on Improving HbA1c Levels in Diabetic Individuals for Study and Control Group throughout Study Period.

\begin{tabular}{lll}
\hline \multirow{2}{*}{ HbA1c Levels at different points of assessment } & Study Group $\mathbf{N}=\mathbf{5 0}$ & Control Group N=50 \\
\cline { 2 - 3 } & Mean \pm SD & Mean \pm SD \\
\hline Baseline assessment (before intervention) & $8.5+4.11$ & $8.19+4.80$ \\
Second assessment (at 3 months of intervention) & $7.60+4.7$ & $7.92+4.31$ \\
Third assessment (at 6 months of intervention) & $7.21+4.61$ & $8.09+4.80$ \\
F - $p$ value & $0.05^{*}$ & $0.91 \mathrm{~ns}$ \\
\hline
\end{tabular}

Table 6. Effect of lifestyle modification on Lipid Profile for study and Control Groups throughout Study period.

\begin{tabular}{llc}
\hline Lipid Profile Variables & Intervention Group N=50 & Control Group N=50 \\
\cline { 3 - 3 } & Mean \pm SD & Mean \pm SD \\
\hline Total cholesterol (mg/dl): Reference range (0.00-200) & & $229.3 \pm 47.8$ \\
Baseline measurement (before intervention) & $233.3 \pm 48.8$ & $224.3 \pm 46.8$ \\
Second measurement (at 3 months of intervention) & $195.4 \pm 43.2$ & $227.3 \pm 46.9$ \\
Third measurement (at 6 months of intervention) & $163.3 \pm 33.2$ & $0.89 \mathrm{~ns}$ \\
F -p value & $0.000 * * *$ & $0.01 * *$ \\
Triglycerides (mg/dl): Reference range (0.00-150) & & $0.001 * * *$ \\
Baseline measurement (before intervention) & $226.0 \pm 45.9$ & $228.0 \pm 46.9$ \\
Second measurement (at 3 months post intervention) & $185.3 \pm 40.2$ & $223.3 \pm 45.1$ \\
Third measurement (at 6 months post intervention) & $121.1 \pm 33.0$ & $220.3 \pm 45.0$ \\
F -p value & $0.001 * * *$ & $0.79 \mathrm{~ns}$ \\
LDL-C (mg/dl): Reference range (100-130) & & $0.71 \mathrm{~ns}$ \\
Baseline measurement (pre intervention) & $151.0 \pm 44.4$ & $0.001 * * *$ \\
Second measurement (at 3 months of intervention) & $139.1 \pm 34.2$ & $0.001 * * *$ \\
\hline
\end{tabular}




\begin{tabular}{lll}
\hline \multirow{2}{*}{ Lipid Profile Variables } & Intervention Group N=50 & Control Group N=50 \\
\cline { 2 - 3 } & Mean \pm SD & Mean \pm SD \\
\hline Third measurement (at 6 months of intervention) & $128.0 \pm 33.0$ & $142.0 \pm 43.0$ \\
F -p value & $0.001 * * *$ & $0.59 \mathrm{~ns}$ \\
HDL-C (mg/dl): Reference range (40-50) & & $0.001^{* * *}$ \\
Baseline measurement (before intervention) & $37.2 \pm 22.0$ & $35.6 \pm 21.00$ \\
Second measurement (at 3 months post intervention) & $40.3 \pm 23.0$ & $38.2 \pm 22.8$ \\
Third measurement (at 6 months of intervention) & $42.2 \pm 21.00$ & $38.9 \pm 22.8$ \\
F -p value & $0.99 \mathrm{~ns}$ & $0.19 \mathrm{~ns}$ \\
\hline
\end{tabular}

$P$ value $*<0.05$ is significant.

$\mathrm{P}$ value $* *<0.001$ is highly significant.

$\mathrm{P}$ value $=\mathrm{ns}$ (non-significant).

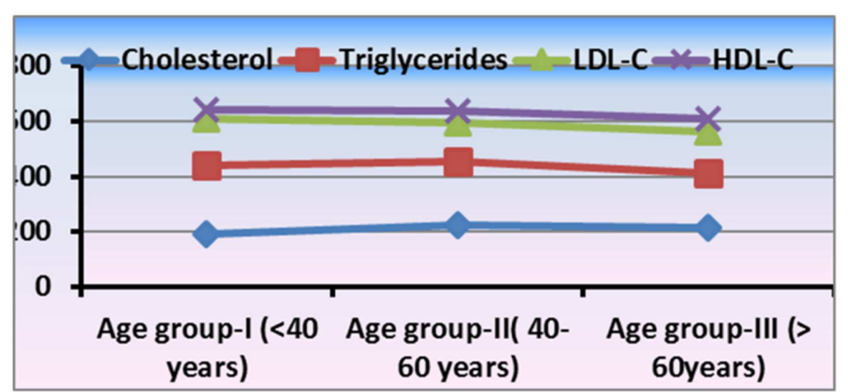

Figure 2. Effect of age on lipid profile in male cardiac patients

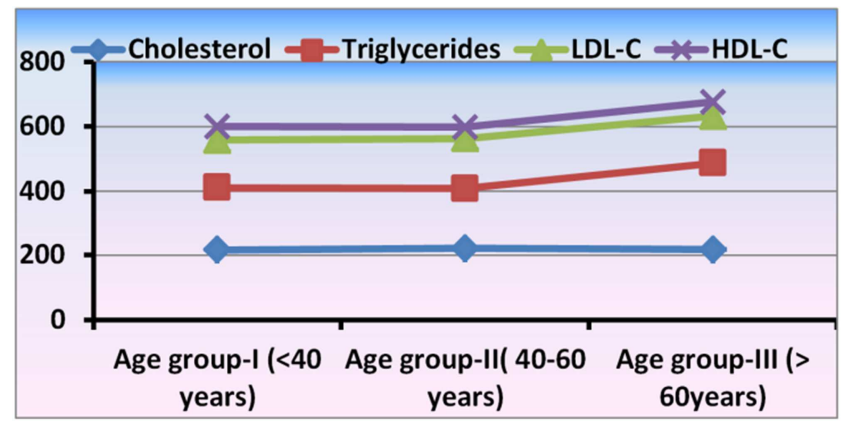

Figure 3. Effect of age on lipid profile in female cardiac patients.

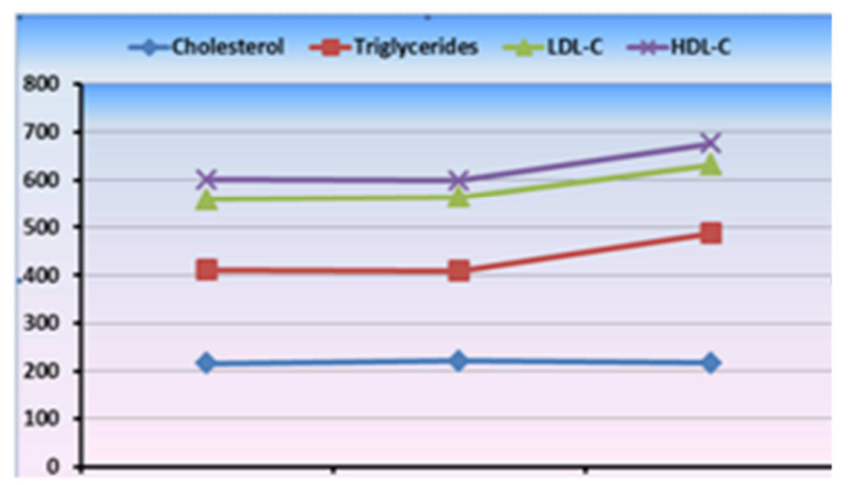

\section{University Secondary Primary}

Figure 4. Effect of educational level on lipid profile in cardiac patients.

\section{Discussion}

Hyperlipidemia is a serious emerging concern all over the world and correlated to sudden cardiac deaths and CVD [29]. The current study aimed to examine the effect of lifestyle modification among those patients on their BMI, BP, and
HbA1c level and lipid profile values. The findings of the current study verifying the hypotheses and proved that using reduction of risk factors has positive effects on BMI, BP, HbA1c level and lipid profile values.

Effective correction of risk factors of cardiovascular diseases is a key component of cardiac rehabilitation and prevention programs, which has proven impact on both the prognosis of patients with CAD [30].

Lifestyle changes and pharmacotherapy, can help lowering the risk of atherosclerotic disease [31, 32].

\section{Abnormal Lipid Profile}

The current study findings reported that there were elevated Triglyceride, Low Density Lipoprotein (LDL), cholesterol and decrease High Density lipoprotein (HLD) in patients of $\mathrm{CHD}$, that was consistent with studies done in Arab countries [3, 33]. That show the abnormal lipid profile remains a strong risk factor of CHD. All of them concluded that patients with CHD have altered lipid profile, with higher levels of TGs, total cholesterol, and LDL and low level of serum HDL; this difference may play a role in the pathophysiology found in Patients with CHD.

Regarding blood glucose level

In current study there was no statistically significant differences between the study and control group regarding the HbA1c level at baseline assessment (before intervention), while there was a statistically significant difference between the both groups at 3 and 6 months of intervention. Results of the current study were consistent with the results of [34] that studied the impact of telemedicine Service for the Secondary Prevention of CAD and concluded that life style modification helped in improving the risk profile in ACS survivors, especially for overweight patients. These also were in accordance with the results of $[35,36]$ who indicated that there was an obvious decreasing in the level of HbAlc after following life style modification. These also were in consistent with the results of [37] who evaluated risk factor control for CAD secondary prevention on 5,034 CAD patients suffering also from diabetes mellitus, they indicated that individual risk factor control was improved compared with baseline. Several studies have revealed that nursing intervention in outpatient clinics is at least as beneficial in achieving goals and actually result in marked improvement in the outcomes, such as a randomized-controlled trial that conducted by [38] who revealed that their designated intervention program improved both SBP and DBP and 
glycemic control through patient self-management education, establishment of an individualized care plan, behavioral goal setting, and close surveillance of patients. A logical explanation of this result is that risk reduction intervention that introduced to those patients by cardiac outpatient nurse and continuous enhancement during follow up sessions on regular bases and they would be provided with some information about their illness and their treatment method among intervention group led to better adherence to diet, and drug therapy prescribed by the doctor, more adaption to physical activity, increased awareness and also changing their bad health behaviors which in turn improve blood glucose level. Therefore this nursing strategy could proceed as a part of health plan for CAD patients. Indeed this preventative nursing strategy provides patients with the opportunity to maintain their education effectively without interruptions, as well as this will empower them to recognize lifestyle changes that need to be addressed while becoming self-efficient. This empowerment will lead to better of glucose control which will improve their clinical outcomes.

\section{Regarding Lipid Profile}

Findings of the current study revealed that mean of total lipid profile scores of studied sample were similar in baseline assessment in both groups. However, the results of this study revealed that implementing risk reduction intervention resulted in improving lipid profile values. It seems that improvement in these results of CAD patients is dependent on behavior change and also lifestyle modification. These results came in accordance with [39].

The mean scores of total lipid profile were highly significantly decreased at 3 months among study subjects patients after implementing risk reduction intervention and continued to be decreased in the 6 months assessment time, with considerable significant difference with subjects in control groups, this positive change in the study group subjects reflected that risk reduction intervention could make change in their lipid profile and showed a considerable difference in its results after the implementing this intervention. In other words, the reductions in the LDL-C level and the TC, HDL-C were greater for intervention patients. After adjustment for baseline differences in blood lipid levels, the study group was also more likely to reach the recommended lipid targets. This positive result was supported the study hypothesis, the clinical impact of risk reduction intervention feedback was high. This was due, in part, to designated risk reduction intervention which mainly based on encouraging patients to adherence to the suggested lifestyle modification protocol including enhancing selfmonitoring and using the support of family and friends. These results came in accordance with recommendation of [40]. This means that that the lifestyle modification has an association and positive impact upon lipid among patients who suffering from hyperlipidemia.

Moreover, the present study results were consistent with many previous studies as [34] who claimed that effective correction of cardiovascular risk factors is a key component of cardiac rehabilitation and secondary prevention programs, which has proven impact on both the prognosis of patients with CAD. Also, this supported by [29] who claimed that both of lifestyle changes and pharmacotherapy, can help reduce the risk of atherosclerotic. Life style modification implies eat heart-healthy foods, exercise on most days of the week and increase physical activity, smoking cessation, weighting lose, and moderation of alcohol intake.

The current results supported by [41] who stated that aerobic exercise decreases cardiovascular disease risk in postmenopausal women and shows potential impact on lipid profile and better quality of life. It also came in accordance with study done by [42] which suggested that improving diet and aerobic fitness will result in a better lipid profile. Also, [40] added that hyperlipidemia can be controlled by weight loss, decreasing dietary fat, regular exercise, and medications. These modalities of treatment decrease TC, LDL, and TG as well as increase the serum level of HDL cholesterol.

This study results were contradicted with [43] who found a non-significant improvement in other lipid profile parameters with exercise was confirmed.

In this study, it was observed that the elevated Triglyceride, Low Density Lipoprotein (LDL), cholesterol and decrease High Density lipoprotein (HLD) in patients of CAD that correspond with $[3,33]$ that shows the abnormal lipid profile remain strong risk factor of CHD. All of them concluded that patients with CHD have altered lipid profile, with higher levels of TGs, total cholesterol, and LDL and low level of serum HDL; this difference may play a role in the pathophysiology found in Patients with CHD.

It is apparent from study findings that, female more than 40 years of age and subjects with primary level of education had significantly the highest mean total lipid profile scores, the older the woman, the higher the elevated lipid profile score with stationary level in cholesterol. Subjects who had primary education had higher abnormal lipid profile score than those who had secondary and university level. Furthermore, the findings of the existing study reported that, there is a strong positive correlation was found significant variations by socio-demographic characteristics.

\section{Regarding $B M I$ and $B P$}

The current risk reduction intervention stressed the importance of weight reduction in order to reduce blood pressure and the body weight and thus reduce the risk of recurrent CVD. The current results revealed a statistically significant difference with the intervention group with a reduction of systolic, diastolic blood pressure as well as loss of weight as manifested by reduction in their BMI. A logical explanation may be pertained to changing diet and increasing physical activity among those patients a step to lifestyle modification where they received advice to do more general daily physical activities. These results came in accordance with [44] who conducted the Euroaction trial of a nurse-led, multidisciplinary hospital programme for CAD patients; they concluded that there were significant improvements in diet and physical activity levels, with three times as many patients achieving the physical activity target in comparison with usual care. 
However, these results were contradicted with [45] study who mentioned that despite the high use of blood pressure lowering medications just over half of patients had achieved the recommended blood pressure goal and lipid control was unsatisfactory in a large proportion of patients. [46, 47] Linked such results to the reasons for the poor lifestyle adherence because of persuading patients to adapt to healthier habits needs skills in behavioral science and time enough to explain the importance. Moreover, and as shown by [48] added that patients have anxiety and depression symptoms after CHD events. Thus, the receptivity to lifestyle advice may be less than optimal immediately after the coronary event, repeating or reinforcing when the patient is in a better condition to accept such counseling is essential.

\section{Conclusions}

Cardiac outpatient department nurses can be utilized lifestyle modifications appropriately as a risk reduction intervention for CAD patients who were suffering from hyperlipidemia. This intervention had an impact upon the clinical outcomes parameters among those patients which in turn will positively impact their quality of life, which is a paramount to the effective intervention for this chronic disease. They should increase their familiarity with exercise programming and dietary protocols to better provide comprehensive preventive and curative lifestyle modifications. In order to effectively combat CAD, they should underscore the necessity of lifestyle modification as a risk reduction intervention and prioritize its use in outpatient department. Implementing such intervention is associated with a measurable improvement in the efficacy of clinical outcomes parameters.

\section{Recommendation}

In the light of the study findings, the following recommendations are suggested:

1. Risk reduction intervention should be integrated to be a part of health plan for CAD patients.

2. Further researches are required to study the factors affecting implementation and utilization of risk reduction intervention.

3. Health care providers must be better trained in risk reduction intervention through their formal and clinical education.

4. Hyperlipidemia among CAD patients may potentially decrease if providers prescribe lifestyle modification program more frequently.

\section{Abbreviation}

Lifestyle modification (LSM) total cholesterol (TC), triglyceride (TG), high-density lipoprotein cholesterol (HDLC) and low-density lipoprotein cholesterol (LDL-C), coronary heart disease (CHD).

\section{References}

[1] Rhee EJ, et al., (2019): 2018 Guidelines for the management of dyslipidemia. Korean J Intern Med. Jul; 34 (4): 723-771. doi: 10.3904/kjim.2019.188. Epub 2019 Jul 1. No PMID: 31272142 .

[2] Wiggins BS, Dixon D, Bellone J, Gasbarro N, Marrs JC, Tran R., (2019): Guidelines in the Management of Dyslipidemia: 2019 Update". J Pharm Pract. Aug 11: 897190019868413. doi: 10.1177/0897190019868413. PMID: 31401932.

[3] El-Moselhy et al., (2018). Coronary Artery Disease among Elderly Egyptian Patients: I. Socio-Demographic, Lifestyle, Psychosocial, Medical, and Biochemical Risk Factors. American Journal of Gerontology and Geriatrics, Volume 1, Issue 2, e1006.

[4] Jellinger PS, et al., (2017): American Association Of Clinical Endocrinologists And American College Of Endocrinology Guidelines For Management Of Dyslipidemia And Prevention Of Cardiovascular Disease. Endocr Pract.; 23 (Suppl 2): 1-87. doi: 10.4158/EP171764.APPGL. PMID: 28437620.

[5] Adhyaru BB, Jacobson TA., (2016): New Cholesterol Guidelines for the Management of Atherosclerotic Cardiovascular Disease Risk: A Comparison of the 2013 American College of Cardiology/American Heart Association Cholesterol Guidelines with the 2014 National Lipid Association Recommendations for Patient-Centered Management of Dyslipidemia. Endocrinol Metab Clin North Am. 2016 Mar; 45 (1): 17-37. doi: 10.1016/j.ecl.2015.09.002. Review. PMID: 26892995.

[6] Berman AN, Blankstein R., (2019) Optimizing Dyslipidemia Management for the Prevention of Cardiovascular Disease: a Focus on Risk Assessment and Therapeutic Options. Curr Cardiol Rep.; 21 (9): 110. doi: 10.1007/s11886-019-1175-z. Review.PMID: 31378838.

[7] World Health Organization, (2012). Cardiovascular diseases (CVDs) Fact sheet no. 317. Available from: http://www.who.int/mediacentre/factsheets/fs317/en/index.ht $\mathrm{ml}$.

[8] United Nations. (2015) Transforming our world: the 2030 agenda for sustainable development.. https://www.un.org/ga/search/ view_doc.asp, symbol=A/RES/70/1\&Lang=E (accessed July 26, 2019).

[9] Roth GA, Abate D, Abate KH, et al. Global, (2018) regional, and national age-sex-specific mortality for 282 causes of death in 195 countries and territories, 1980-2017: a systematic analysis for the Global Burden of Disease Study 2017. Lancet; 392: $1736-88$.

[10] WHO. HEARTS technical package. (2019). https://www.who.int/ publications-detail/hearts-technicalpackage.

[11] Tips for Healthy lifestyle changes (modification) and Wellness (2017). https://www.jotscroll.com > forums > posts > healthylifestyle-change modification. American college of life style medicine https://lifestylemedicine.org/ACLM/Education/Board.

[12] NICE Pathway, Cardio Vascular Disease Prevention, (2019) http://pathways.nice.org.uk/pathways/cardiovascular-diseaseprevention. 
[13] Wilson PW., Polonsky TS., Miedema MD., Khera A., Kosinski AS., Kuvin JT., (2019) Systematic Review for the Guideline on the Management of Blood Cholesterol: A Report of the American College of Cardiology/American Heart Association Task Force on Clinical Practice Guidelines. J Am Coll Cardiol. Jun 25; 73 (24): 3210-3227. doi: 10.1016/j.jacc..11.004. PMID: 30423394.

[14] Kundu, D., Saikia, M. \& Paul, T. (2017). Study of the Correlation between Total Lipid Profile and Glycosylated Hemoglobin Among the Indigenous Population of Guwahati. Int. J. Life. Sci. Scienti. Res, 3, 1175-1180.

[15] Singh, R. J., Kohli, K., Gupta, A. K., \& Gupta, S. (2018). Hyperlipidemia: Treatment Modality And Busting Myths Of Dietary Approach. World Journal of Pharmaceutical ResearchSJIF Volume 7, Issue 16, 395-406.

[16] Arshad H, Iftikhar A, Waqar K and Fatima Y., (2019) Correlation between Body Mass Index and Lipid Profile in patients with Type 2 Diabetes attending a tertiary care hospital in Peshawar, Pak J Med Sci. May-Jun; 35 (3): 591-597. doi: 10.12669/pjms.35.3.7

[17] Ettehad D, Emdin CA, Kiran A, et al., (2016) Blood pressure lowering for prevention of cardiovascular disease and death: a systematic review and meta- analysis. Lancet; 387: 957967.

[18] Patnode CD, Evans CV, Senger CA, Redmond N, Lin JS. (2019). Behavioral Counseling to Promote a Healthful Diet and Physical Activity for Cardiovascular Disease Prevention in Adults Without Known Cardiovascular Disease Risk Factors: Updated Systematic Review for the U.S. Preventive Services Task Force [Internet].

[19] Bei, Y., Zhou, Q., Sun, Q. \& Xiao, J. (2015). Exercise as a platform for pharmacotherapy development in cardiac diseases. Current pharmaceutical design, 21, 4409-4416.

[20] Lucas, J.-M. \& Kozlowski, K. F. (2019). The Underutilization of Lifestyle Modifications in Primary Care Medicine. Exercise Medicine, 3, 3 .

[21] Sanin V, Koenig W. (2019) Therapy of Hypercholesterolemia in Primary Prevention. Dtsch Med Wochenschr. Mar; 144 (5): 322-328. doi: 10.1055/a-0657-1668. Epub. 5. Review. German. PMID: 30836403.

[22] Yiyi Zhang et al., (2019) Associations of Blood Pressure and Cholesterol Levels During Young Adulthood With Later Cardiovascular EventsJournal of the American College of Cardiology Volume 74, Issue 3, DOI: 10.1016/j.jacc.2019.03.529.

[23] Arnett DK, et al., (2019) ACC/AHA Guideline on the Primary Prevention of Cardiovascular Disease: A Report of the American College of Cardiology/American Heart Association Task Force on Clinical Practice Guidelines. Circulation. 2019 Sep 10; 140 (11): e596-e646.

[24] Lloyd-Jones DM, et al., (2017) Focused Update of the 2016 ACC Expert Consensus Decision Pathway on the Role of Non-Statin Therapies for LDL-Cholesterol Lowering in the Management of Atherosclerotic Cardiovascular Disease Risk: A Report of the American College of Cardiology Task Force on Expert Consensus Decision Pathways. J Am Coll Cardiol. 2017 Oct 3; 70 (14): 1785-1822. doi: 10.1016/j.jacc.2017.07.745. Epub 2017 Sep 5. PMID: 28886926.
[25] Chikwere, P. \& Annan, R. A. (2016). Dietary habit and other lifestyles and serum lipid profile of type 2 diabetes patients: a systematic review. Nutrition \& Food Science, 46, 161-170.

[26] Weber C, Badimon L, Mach F, van der Vorst EPC. (2017) Therapeutic strategies for atherosclerosis and atherothrombosis: past, present and future. Thromb Haemost; 117: 1258-1264. Google Scholar

[27] Ghanwat, G. H. \& Sontakke, A. V. (2018). Effect of Vitamin C Supplementation on Anthropometric Measurements, Lipid Profile and Atherogenic Indices in Obese and Non Obese Individuals. Journal of Clinical \& Diagnostic Research, 12.

[28] Kelsey and Fleiss (2010). Methods in observational Epidemiology 2nd Edition, Statistical Methods for Rates and Proportion, formulas 3.18 and 19. Avaliable at: Epi website (Open Source Statistics for Public Health): http://www.openepi.com/SampleSize/SSCohort.htm.

[29] Hartz, J. \& Clauss, S. (2017). Treatment Strategies for Hypercholesterolemia. Current pediatric reviews, 13, 243-254.

[30] Lubinskaya E., Protasov, E., and. Demchenko, E. (2016): The level of cardiovascular risk factors correction in patient undergoing myocardial revascularization, Symposium of the International Atherosclerosis Society June 2-4, 2016, St. Petersburg, Russia.

[31] TURAN, T. N., et al., (2018). Relationship between risk factor control and compliance with a lifestyle modification program in the stenting aggressive medical management for prevention of recurrent stroke in intracranial stenosis trial. Journal of Stroke and Cerebrovascular Diseases, 27, 801-805.

[32] Abess et al. (2017): Lipid Profile and Coronary Heart Disease. International journal of pharmacy \& Pharmaceutical Research (ISSN 2349-7203), Vol.: 11, Issue: 1.

[33] Al-Hassan, Y. T. \& Fabella, E. L. (2017). Lipid profile analysis of patients in a saudi university clinic. Public Health International, 2, 89-95.

[34] Blasco, A., et al., (2012). Evaluation of a telemedicine service for the secondary prevention of coronary artery disease. Journal of cardiopulmonary rehabilitation and prevention, 32 (1), 25-31.

[35] Del Prato S, Nicolucci A, Lovagnini-Scher AC, Turco S, Leotta S, Vespasiani G: (2012) Telecare Provides comparable efficacy to conventional self-monitored blood glucose in patients with type 2 diabetes titrating one injection of insulin glulisine-the Eleonor study. Diabetes Technol Ther, 14: 175182. | Article | PubMed.

[36] Gungar T, Karadakovan and Saygili F (2013). The use of videophone technology (telenursing) in the glycaemic control of diabetic patients: a randomized controlled trial. Journal of Diabetes Research \& Clinical Metabolism, ISSN 2050-0866

[37] Farkouh, M. E., et al., (2013). Risk factor control for coronary artery disease secondary prevention in large randomized trials. Journal of the American College of Cardiology, 61 (15), 16071615 .

[38] Beyazıt, E., \& Mollaoğlu, M. (2011). Investigation of effect on glycosylated hemoglobin, blood pressure, and body mass index of diabetes intensive education program in patients with type 2 diabetes mellitus. American journal of men's health, 5 (4), 351-357. 
[39] Ibrahim, M. M., Ibrahim, A., Shaheen, K. \& Nour, M. A. (2013). Lipid profile in Egyptian patients with coronary artery disease. The Egyptian Heart Journal, 65, 79-85.

[40] Párraga-Martínez, I., et al., (2018). Efficacy of a Combined Strategy to Improve Low-density Lipoprotein Cholesterol Control among Patients With Hypercholesterolemia: A Randomized Clinical Trial. Revista Española de Cardiología (English Edition), 71 (1), 33-41.

[41] Akwa, L. G., et al., (2017). Lipid profile, cardiorespiratory function and quality of life of postmenopausal women improves with aerobic exercise. Journal of Human Sport and $\begin{array}{lllll}\text { Exercise, } & 12 & \text { (3), 698-709. doi: }\end{array}$ https://doi.org/10.14198/jhse.2017.123.14.

[42] Wadden, T. A., Webb, V. L., Moran, C. H. \& Bailer, B. A., (2012). Lifestyle modification for obesity: new developments in diet, physical activity, and behavior therapy. Circulation, $125,1157-1170$.

[43] Cugnetto, M. L., Saab, P. G., Llabre, M. M., Goldberg, R., McCalla, J. R., \& Schneiderman, N. (2008). Lifestyle factors, body mass index, and lipid profile in adolescents. Journal of pediatric psychology, 33 (7), 761-771.

[44] Wood DA, Kotseva K, Connolly S, et al.; (2008) on behalf of
Euroaction Study Group. Nurse-coordinated multidisciplinary, family-based cardiovascular disease prevention programme (Euroaction) for patients with coronary heart disease and asymptomatic individuals at high risk of cardiovascular disease: A paired, cluster-randomised controlled trial. Lancet; 371: 1999-2012.

[45] Rigotti NA, Munafo MR and Stead LF., (2008) Smoking cessation interventions for hospitalized smokers: A systematic review. Arch Intern Med; 168: 1950-1960.

[46] Ammar, T., (2015). Effects of aerobic exercise on blood pressure and lipids in overweight hypertensive postmenopausal women. Journal of Exercise Rehabilitation, 11 (3), 145-150. http://doi.org/10.12965/jer.150204.

[47] Kotseva, K., \& Euroaspire Investigators. (2017). The Euroaspire Surveys: lessons learned in cardiovascular disease prevention. Cardiovascular diagnosis and therapy, 7 (6), 633.

[48] Pogosova N., Kotseva K., De Bacquer D., et al., (2017) Psychosocial risk factors in relation to other cardiovascular risk factors in coronary heart disease: Results from the Euroaspire IV Survey. A Registry From The European Society of Cardiology, on Behalf of The Euroaspire Investigators. Eur J Prev Cardiol. Epub ahead of print 23. DOI: 10.1177/ 2047487317711334 